\title{
Estimating Muscular Activity of the Quadriceps Femoris Muscles by Measuring the Change in Shape
}

\author{
Hielscher J. ${ }^{1}$, Meiss $T .{ }^{1}$, Block J. ${ }^{2}$, Werthschützky R. ${ }^{1}$ \\ ${ }^{1}$ Measurement and Sensor Technology Lab., Institute for EMK, Technical University Darmstadt, \\ Darmstadt, Germany, j.hielscher@emk.tu-darmstadt.de \\ ${ }^{2}$ Motionlab, Department Orthopedic Surgery, Heidelberg University Clinics, Heidelberg, Germany
}

\begin{abstract}
:
This paper introduces a novel sensor concept for measuring muscular activity assessing the change of the skin surface curvature. The contraction of the rectus femoris muscle is expected to provide information on the user's intention to rise from a chair. This information will be used for the control of a motorized lower limb orthosis to improve the quality of life of people suffering from muscle weakness. Electromyography is widely used for measuring muscular activity, but is affected by sweat and aging effects of the glue used to attach the electrodes. Furthermore, the necessity of glued, skin contacted electrodes reduces the ergonomic qualities. The sensor presented is designed for long-term use in an every-day environment. It consists of a thin metal stripe placed over the muscle. Deformation of the muscle during contraction causes a bending of the stripe. The generated surface strain can be measured with strain gauges. For the application around the user's thigh the stripe is incorporated in an elastic textile cuff. The capability of the system is evaluated in isometric exercises and sit-to-stand tasks, each compared to simultaneously recorded EMG data. Contraction and relaxation of the muscle were recognized in the signal curve. Isometric exercises revealed a higher sensitivity of the sensor for low muscular activity. During sit-to-stand tasks the signal curve showed a high similarity to the calculated knee torque. Future developments will focus on the reduction of soft tissue influence and muscle cross-talk.
\end{abstract}

Key words: Muscle Sensor, EMG, Muscular Activity, Sit-to-Stand, Orthosis

\section{Introduction}

Reduced mobility of the elderly is often based on muscle weakness and fatigue [1, 2]. People concerned suffer from a reduced participation in activities of daily living and less independence, leading to a lower quality of life. This is not only a problem for the individual, but also for the society at large. Therefore, the development of devices to maintain or improve mobility is a desirable research goal. One approach to pursue this aim is a motorized orthosis, equipped with actuators to drive the knee joint. By providing an external power, the device helps to master the key tasks of everyday life such as standing up from a chair, stepping into the train, stair climbing, etc. The device thereby does not supersede the effort of the user, but compensates the muscular deficiency. One crucial problem in the development of motorized assisting devices is the lack of knowledge about the user's intention for the proper and safe control of the actuator. Since the user's lower limb muscles are weak but not paralyzed, the detection of muscular activity is expected to provide decisive information. Electromyography (EMG) is widely used for measuring muscular activity in clinical environments, yet also for assisting devices $[3,4]$. However, EMG is prone to electromagnetic disturbances, influence of sweat on the skin-electrode-interface and aging effects of the glue used to attach the electrodes. The signal depends on how the user places the electrodes and varies with every new application. Furthermore, the necessity of glued, skin contacted electrodes reduces the ergonomic qualities. Thus, EMG is inappropriate for long-term usage in an assisting device. This paper reports the research of a novel approach for estimating muscular activity by measuring the change of 
the cross sectional shape of the muscle belly during contraction emerging on the skin surface above the muscle. The study focuses on the Sit-To-Stand movement (STS), chosen for its key importance in everyday life. It is a challenging task for the elderly due to its high demands in terms of muscle power and balance control [5].

\section{Physiological considerations}

To estimate the user's intention of rising up from a chair by the means of muscular activity, involved muscles providing characteristic and repeatable attributes must be identified. In studies investigating activation patterns of the lower limb muscles during the STS movement using EMG, the largest increase of activity was found for the quadriceps femoris muscles (QFM) [6, 7]. This is apparent since the QFM are responsible for knee extension, which is (besides the extension of the hip) the central movement in the erection of the body from a seated position. Consisting of the four synergistic heads musculus vastus medialis, $\mathrm{m}$. vastus intermedius, $m$. vastus lateralis and $m$. rectus femoris (MRF), the QFM is large in size and hence providing extensive change in shape. Based on these facts, the QFM were chosen for the assessment of the user's intention.

Muscle contractions are generated by the filaments in every muscle fiber sliding against each other. Thus, the contraction causes an increase of the muscle cross sectional area. Studies have shown a correlation between the knee extension force and the cross sectional area of the quadriceps femoris muscles [8, 9]. Additionally, due to the spindle-like shape of the muscle the increasing tension during contraction generates a force on muscle fibers in the exterior regions towards the axis. The cross section profile of the muscle changes during contraction from flat oval-shape towards round shape. These effects result in a bulging of the QFM and hence, regarding the cross sectional profile of the thigh, a higher skin surface curvature.

\section{Sensing Device}

To measure changes of the skin surface curvature, a bending sensor was designed (Fig.1). It consists of a spring steel stripe $\left(100 \times 17 \times 0.2 \mathrm{~mm}^{3}\right)$ placed over the muscle. Deformation of the muscle during contraction causes a bending of the stripe. The generated surface strain can be measured with strain gauges. Spring steel features high flexibility and yield strength providing a low risk of plastic deformation. To obtain a high bending and a low backlash a thin stripe is beneficial. On the other hand, a large strain on the surface and thus a high resolution of the sensor can be achieved by using a thick stripe. Tests with several stripes revealed the best tradeoff with a thickness of $0.2 \mathrm{~mm}$ and a width of $17 \mathrm{~mm}$. Two metallic strain gauges (HBM, SG/C 1-LC11-10, 350 Ohms) were applied in parallel alongside the stripe. One gauge is used for the measurement, the second is redundant for compensation in case of failure. The steel stripe is incorporated in a cuff made of elastic fabric, providing a safe and comfortable fit.

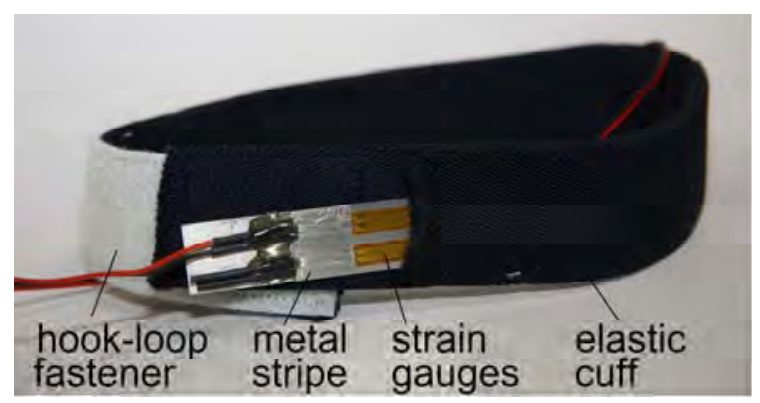

Fig. 1. Bending Sensor with elastic cuff for measuring the deformation of the musculus rectus femoris during contraction.

\section{Experiments}

The suitability of the bending sensor for detecting the QFM activity is evaluated in two tests. First, the signal of the sensor is compared to the EMG signal of the MRF and the intensity of the muscular contraction in isometric condition. In the second test the sensor signal is evaluated in comparison to EMG and the knee torque derived by the means of inverse dynamics during sit-to-stand movements.

\section{Setup}

The cuff was placed around the thigh with the stripe over the belly centre of the MRF, where the deformation is expected to be largest. The sensor stripe is aligned in perpendicular direction to the femur axis. The strain gauge was connected to a bridge amplifier (Kistler 5271, quarter bridge configuration, bridge excitation $U_{e}=5 \mathrm{~V}, \quad$ amplification $\mathrm{V}=200$, low pass filter cut-off frequency $f_{c}=10 \mathrm{~Hz}$ ). The surface EMG of the according muscle and the signal of the bending sensor were acquired simultaneously with a data acquisition board (sampling rate $f_{s}=1080 \mathrm{~Hz}$, resolution $16 \mathrm{bit}$ ). The EMG signal was conditioned by removing the offset and applying a moving average filter with $500 \mathrm{~ms}$ and $100 \mathrm{~ms}$ window length for isometric and STS-tests, respectively. Finally, both signals were scaled to 1 . 


\section{Isometric Exercises}

The subjects were asked to sit on a bench without back and armrests with an initial position of $90^{\circ}$ flexion in knee and hip. The extension of the right leg was suppressed with a rigid rope fixed with a cuff on the subject's ankle. The arm position was consistently on the chest. Due to the compliance of the cuff and leg tissue, a small horizontal displacement of the foot must be enabled. This is achieved by placing the foot on a creeper dolly. Three healthy volunteers (1f, $2 \mathrm{~m}$, age: median $=29$ (range $=27-34) ;$ height: $1.77 \mathrm{~m}$ $(1.68 \mathrm{~m}-1.80 \mathrm{~m}) ; \quad$ mass $72.5 \mathrm{~kg} \quad(55 \mathrm{~kg}-$ $84 \mathrm{~kg})$ ) were requested to perform six trials. In each trial the subject was asked to exert a predefined force level $(0 \mathrm{~N}, 20 \mathrm{~N}, 40 \mathrm{~N}, 60 \mathrm{~N}$, $80 \mathrm{~N}, 100 \mathrm{~N}$ ) against the rope by extending his shank. Each level should be held for five seconds. At higher forces the subjects lost seat contact and the task could not be performed properly. The force was measured with a force sensor and instantaneously displayed, to provide a visual real time feedback to the subject. The force signal was acquired simultaneously to the signals from EMG and the bending sensor. Afterwards, the knee torque was calculated by multiplying the exerted force by the length of the shank measured from the knee to the ankle (lateral malleolus).

\section{Sit-to-Stand Exercises}

Four volunteers (1f, $3 \mathrm{~m}$; age: median $=29.5$ (range $=27-34)$; height: $1.76 \mathrm{~m} \quad(1.68 \mathrm{~m}-$ $1.80 \mathrm{~m}) ; \quad$ mass $71.25 \mathrm{~kg} \quad(55 \mathrm{~kg}-84 \mathrm{~kg}))$ performed 15 STS movements each. The subjects were requested to rise in self-selected velocity and in straightforward direction from a bench without back and armrests. Seat height was adjusted for $90^{\circ}$ knee flexion, the arm position was consistently on the chest. The seat-off was detected for later visualization purposes by placing the bench on a force plate. Additionally, the kinetic and kinematic data was acquired from one of the volunteers using force plates under each foot (Kistler) and a 3 dimensional motion capture system (Vicon), respectively. The knee torque was computed using the Vicon Plug-in-Gait model $[10,11]$.

\section{Results}

Isometric Exercises

A total of 16 trials were examined ( 3 subjects, 6 trials, 2 corrupted data sets). In 14 out of 16 trials the signal of the bending sensor showed a distinct peak at the moment of the first muscular contraction. The relaxation of the muscle was detected in every trial. Different levels of contraction could be clearly distinguished in 8 trials. The bending sensor showed a nonlinear sensitivity to the muscle contraction. Weak muscle contractions induced a large change of amplitude.

Tab. 1: Results of the isometric tests comparing electromyography (EMG) to the novel bending sensor (BS)

\begin{tabular}{|l|c|c|}
\hline & EMG & BS \\
\hline $\begin{array}{l}\text { Distinct amplitude in the } \\
\text { moment of first } \\
\text { contraction }\end{array}$ & $16 / 16$ & $14 / 16$ \\
\hline $\begin{array}{l}\text { Distinct amplitude in the } \\
\text { moment of relaxation }\end{array}$ & $16 / 16$ & $16 / 16$ \\
\hline $\begin{array}{l}\text { Distinguishable levels of } \\
\text { contraction }\end{array}$ & $14 / 16$ & $8 / 16$ \\
\hline
\end{tabular}

Towards strong contractions lower changes were noticed, leading to a merging of the amplitude in some trials. In the EMG data both, the contraction and relaxation, could be recognized in every trial. Different levels of contraction were distinguishable in 14 out of 16 trials. Hereby the higher dispersion towards high muscular activity impairs the certain distinction of the different contraction levels in the data.

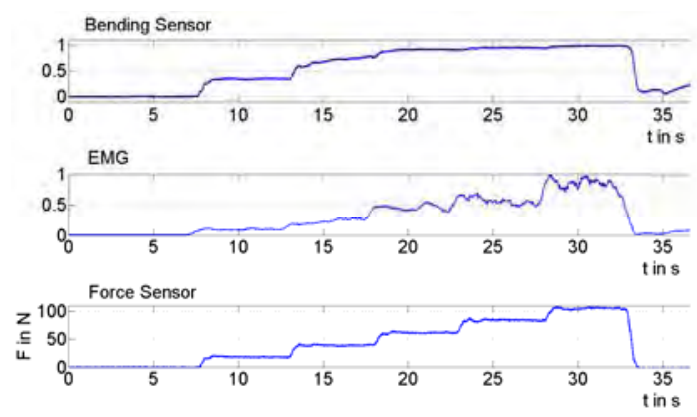

Fig. 2. Signal of the bending sensor compared to EMG and exerted force during isometric exercises.

Sit-to-Stand Exercises

A total of 59 trials were examined (4 subjects, 15 trials, 1 corrupted data set). In 58 out of 59 trials the signal of the bending sensor showed a distinct rise in the moment of the muscle contraction to initiate the chair raise (Fig. 3, mark 1). Compared to the calculated knee torque the signal had a high similarity during the movement. In 22 trials the signal showed a rise of muscular activity before and after the movement without the presence of EMG activity (Fig. 3, mark 2). Thereby the amplitude reached up to $50 \%$ of the maximum value. An offset remained occasionally ( 5 trials) after the raise. 


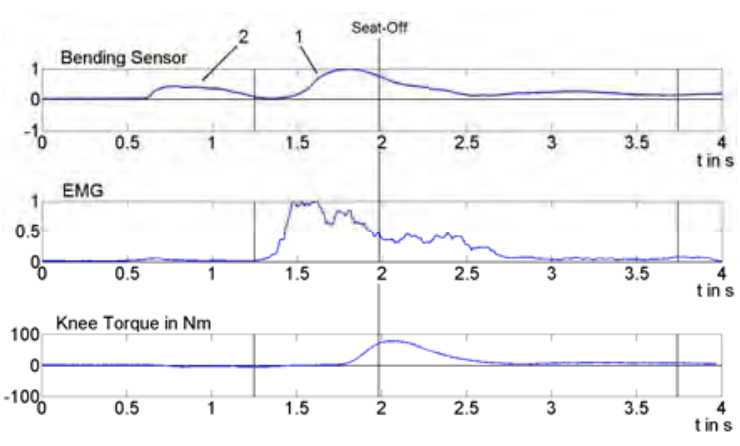

Fig. 3. Signal of the bending sensor compared to EMG and calculated knee torque (inverse dynamics) during STS exercises.

\section{Discussion}

Since the signal provides a reliable increase when the MRF is contracted and a drop when relaxed, the bending sensor detects activation at least as binary signal. This information can be used for a motorized assistive device to start and stop the support. The described amplitudes in times without EMG activity are likely due to muscle cross-talk of the antagonistic knee flexors, since they seem to appear when the subject intuitively pulls its feet under the body to shift its weight in front. Additionally the movement of soft tissue, such as fat and fascia, reduces the deformation on the skin surface and allows skin movement in relation to the muscle. The nonlinear sensitivity to the muscle contraction impedes the resolution of muscular activity at high exerted forces. This yields from the changes of the muscle shape, which by nature turns out smaller at higher activation levels. However, under laboratory conditions the resolution of small changes with the novel bending sensor are possible. The similarity of the signal compared to the knee torque calculated by means of inverse dynamics is promising. The application of the sensor cuff is quick and easy and provides comfortable use. This is an important requirement for the utilization in an assistive device for the elderly to support mobility in daily living. Future developments will focus on the reduction of soft tissue influence and muscle cross-talk.

\section{References}

[1] M. Vanoncini, J. Block, S. Wolf, 3D Analysis of the Sit-to-Stand Movement to Guide the Design of Motorized Orthoses, ISB (2011)

[2] E. Bassey, M. Fiatarone, E. O'Neill, M. Kelly, W. Evans, L. Lipsitz, Leg Extensor Power and Functional Performance in Very Old Men and Women, Clinical Science 82, 321-327 (1992)

[3] D. Andreasen, S.K. Allen, D. Backus, Exoskeleton with EMG Based Active Assistance for Rehabilitation, IEEE Transactions on Robotics 24, 333-336 (2005)
[4] A. Dollar, H. Herr, Lower Extremity Exoskeletons and Active Orthoses: Challenges and State-ofthe-Art, International Conference on Rehabilitation Robotics 24, 144-158 (2008)

[5] M. Lomaglio, J. Eng, Muscle Strength and Weight-Bearing Symmetry Relate to Sit-to-Stand Performance in Individuals with Stroke, Gait and Posture 22, 126-131 (2005)

[6] P. Millington, B. Myklebust, G. Shambes, Biomechanical Analysis of the Sit-to-Stand Motion in Elderly Persons, Arch. Phys. Med. Rehab. 73, 609-617 (1992)

[7] M. Roebroeck, C. Doorenbosch, J. Harlaar, R. Jacobs, G. Lankhorst, Biomechanics and Muscular Activity during Sit-to-Stand Transfer, Clin. Biomech. 9, 235-244 (1994)

[8] Y. Takai, M. Ohta, R. Akagi, H. Kanehisa, Y. Kawakami, T. Fukunaga, Sit-to-stand Test to Evaluate Knee Extensor Muscle Size and Strength in the Elderly: A Novel Approach, Journal of Physiological Anthropology 28, 123128 (2009)

[9] K. Häkkinen, A. Häkkinen, Muscle CrossSectional Area, Force Production and Relaxation Characteristics in Women at Different Ages, European Journal of Applied Physiology 62, 410414 (1991)

[10] M. Kadaba, H. Ramakrishnan, M. Wootten, Measurement of Lower Extremity Kinematics during Level Walking, Journal of Orthopaedic Research 8, 383-392 (1990).

[11] R. Davis, S. Õunpuu, D. Tyburski, J. Gage, A Gait Analysis Data Collection and Reduction Technique, Human Movement science 10, 575578 (1991) 